\title{
Identification and Characteristics of Co-isolation of Multiple Nontuberculous Mycobacteria
}

\author{
Masato Asaoka, Eri Hagiwara, Satoshi Etori, Katsuyuki Higa, Satoshi Ikeda, \\ Akimasa Sekine, Hideya Kitamura, Tomohisa Baba, Shigeru Komatsu and Takashi Ogura
}

\begin{abstract}
:
Objective Although multiple nontuberculous mycobacteria (NTM) species can be isolated from the same patient, little has been reported on co-isolation. We clarified the trends and characteristics of the co-isolation of multiple NTM species.

Methods To collect data on multiple NTM isolation, we first extracted all patients who visited our hospital from 2006 through 2015 with a diagnosis of NTM lung diseases other than Mycobacterium avium complex (MAC) and then reviewed their medical records to evaluate the co-isolation of multiple NTM species.

Results Of 213 patients with non-MAC lung disease, the most common NTM species was M. gordonae (32\%), followed by M. kansasii (20\%) and M. abscessus (14\%). Non-MAC NTM lung disease tended to be associated with middle age with a low body mass index and male predominance. Multiple NTM species were isolated from $55(26 \%)$ of the 213 patients. The clinical characteristics associated with multiple NTM species isolation included female predominance, never smokers and the absence of cavity lesions in the lungs. The highest co-isolation rate was observed in patients with M. gordonae isolation (30\%), followed by M. furtuitum isolation (26\%) and M. abscessus isolation (20\%). Only MAC was isolated when co-isolated with M. abscessus. Among M. szulgai, M. peregrinum and M. terrae isolation, no other NTM species were detected.

Conclusion Co-isolation of multiple NTM species was not uncommon, with $26 \%$ of patients with nonMAC NTM lung diseases showing co-isolation with multiple NTM species. Each NTM species had distinct characteristics in terms of co-isolation.
\end{abstract}

Key words: multiple isolation, nontuberculous mycobacteria, Mycobacterium avium complex, co-infection

(Intern Med 60: 3213-3219, 2021)

(DOI: 10.2169/internalmedicine.5300-20)

\section{Introduction}

The prevalence of nontuberculous mycobacteria (NTM) lung disease is steadily increasing worldwide, especially in industrialized countries, and is recognized as a socially important disease (1). In addition, the number of isolated NTM species has also been increasing rapidly, with more than 150 species identified so far (2). Among them, Mycobacterium avium complex (MAC) is the most common pathogen causing pulmonary NTM infections in many countries, including Japan.

At present, there is an increased focus on the epidemiology of NTM lung disease. A recent large study in Japan re- vealed detailed data on MAC lung disease and the prevalence of non-MAC NTM lung disease (3). According to that study, the prevalence of non-MAC NTM has been steadily increasing (4). However, studies on non-MAC lung disease have not been large enough to allow for the estimation of the prevalence rate. In addition, the treatment outcome of non-MAC NTM lung disease tends to be unsatisfactory due to the lack of studies on such entities (5). Consequently, real-world data on the prevalence of non-MAC NTM isolation are desperately needed in order to clarify NTM species' diversity and assess different therapeutic options.

Some authors have reported the isolation of multiple NTM species in the same patient and co-infections with other NTM species during or after treatment of the initial

Division of Pulmonary Medicine, Department of Respiratory Medicine, Kanagawa Cardiovascular and Respiratory Center, Japan 
NTM species $(6,7)$. We sometimes identify different NTM species from those isolated at the diagnosis in the same patient by conducting regular mycobacterial sputum culture during follow-up. However, studies on the detection of multiple NTM isolates are limited, and the clinical significance of such detection remains unclear.

The present study assessed the prevalence of NTM isolates other than MAC and evaluated the patterns of multiple NTM species isolates in the same patient.

\section{Materials and Methods}

\section{Patients}

To collect data on patterns of multiple NTM co-isolation, we retrospectively extracted data for patients from whom non-MAC NTM were isolated in respiratory specimens at least twice between January 2006 and December 2015. All patients had abnormal lung shadows. The clinical presentation and backgrounds were collected from the medical records.

\section{Microbiological examinations}

Expectorated or induced sputum specimens, and bronchial washings in a few cases were processed in our institution using standard decontamination, fluorochrome microscopy and liquid media culture using MIGT ${ }^{\mathrm{TM}}$ medium (Mycobacteria Growth Indicator Tube; BD, Sparks, USA). We performed species identification tests on all positive cultures using the same biochemical tests.

Patients were classified as "multiple" or "single species isolators". Multiple isolators were defined as patients from whom different NTM species were isolated at least twice. Patterns of multiple NTM species isolation were classified as follows: (i) simultaneous isolation of multiple NTM species $(A+B \rightarrow A+B)$, (ii) alternate isolation of multiple NTM species $(\mathrm{A} \rightarrow \mathrm{B} \rightarrow \mathrm{A} \rightarrow \mathrm{B}$ ) or (iii) disappearance of the initially isolated NTM species and detection of a different NTM species $(A \rightarrow A \rightarrow B \rightarrow B)$. We included all patterns of multiple species isolation.

\section{Data analyses}

Data were analyzed using the SPSS software program (version 26; IBM SPSS, Chicago, USA). All data were presented as the mean \pm standard deviation (SD) if normally distributed or median [interquartile range (IQR)] if nonparametric. Parametric variables were compared using $t$ tests (paired or independent), and nonparametric variables were compared with the Mann-Whitney U test (unrelated) or Wilcoxon's signed rank test (related). Categorical variables were analyzed using the chi-square test. Probability values less than 0.05 were considered statically significant. All probability values were two-sided.

\section{Results}

\section{Clinical background characteristics of patients from whom NTM other than MAC were isolated two or more times}

Two hundred and thirteen patients were included in this study. Twelve NTM species were isolated. Among the 213 patients, $M$. gordonae was the most prevalent strain isolated, followed by M. kansasii, M. abscessus, M. fortuitum and $M$. chelonae (Figure). The patients' median age was 68 (IQR 60-76) years old, and 131 patients $(61.5 \%)$ were men. The median body mass index was $19.6 \pm 2.4 \mathrm{~kg} / \mathrm{m}^{2}$. One hundred and sixty patients $(61 \%)$ had comorbidities, including cancer, interstitial lung disease and diabetes, all of which might increase the likelihood of NTM infection. Eighty-seven $(40.8 \%)$ patients were treated with antibacterial agents (Table 1). The regimens used included not only multidrug regimens for each mycobacterial infection but also long-term, low-dose erythromycin monotherapy. None of the included patients underwent adjuvant lung resection surgery.

Among the patients in whom $M$. gordonae was isolated, 40 were men $(58.0 \%)$, and $49(71.0 \%)$ had at least 1 comorbidity. Ten patients $(14.5 \%)$ had a cavity lung lesion.

\section{Co-isolation of two or more NTM species from same patient}

Multiple NTM species were isolated in 55 of the 213 patients $(26 \%)$. The median age of these patients was 68 (range: 22-85) years old, and 34 (61.8\%) were women. Although patients with non-MAC NTM lung disease were enrolled in our study, 47 of the 55 patients $(85 \%)$ were subsequently confirmed to have MAC.

We evaluated the prevalence of multiple NTM species isolates by the original NTM species (Table 2). For M. gordonae, which was most common species, co-isolation of multiple NTM species was found in 24 (35\%) of 69 patients. Among these 24 patients, MAC was isolated in 21. While the second-most common species to be isolated was M. kansasii (42 patients), multiple NTM isolates were observed in only $12 \%(\mathrm{n}=5)$ of these patients. No other species were co-isolated among the patients with M. szulgai, M. peregrinum or $M$. terrae. Seven patients (23\%) with $M$. $a b$ scessus were found to be harboring multiple NTM species in which MAC was exclusively isolated.

\section{Patterns of multiple NTM isolation from same pa- tient}

Table 3 shows the co-isolation patterns in all 55 patients. Among these patients, three patterns of multiple NTM isolation were identified: (i) simultaneous isolation of multiple NTM species, (ii) alternate isolation of multiple NTM species and (iii) disappearance of the initially isolated NTM species and detection of a different NTM species. Among the 55 patients, simultaneous isolation of multiple NTM spe- 


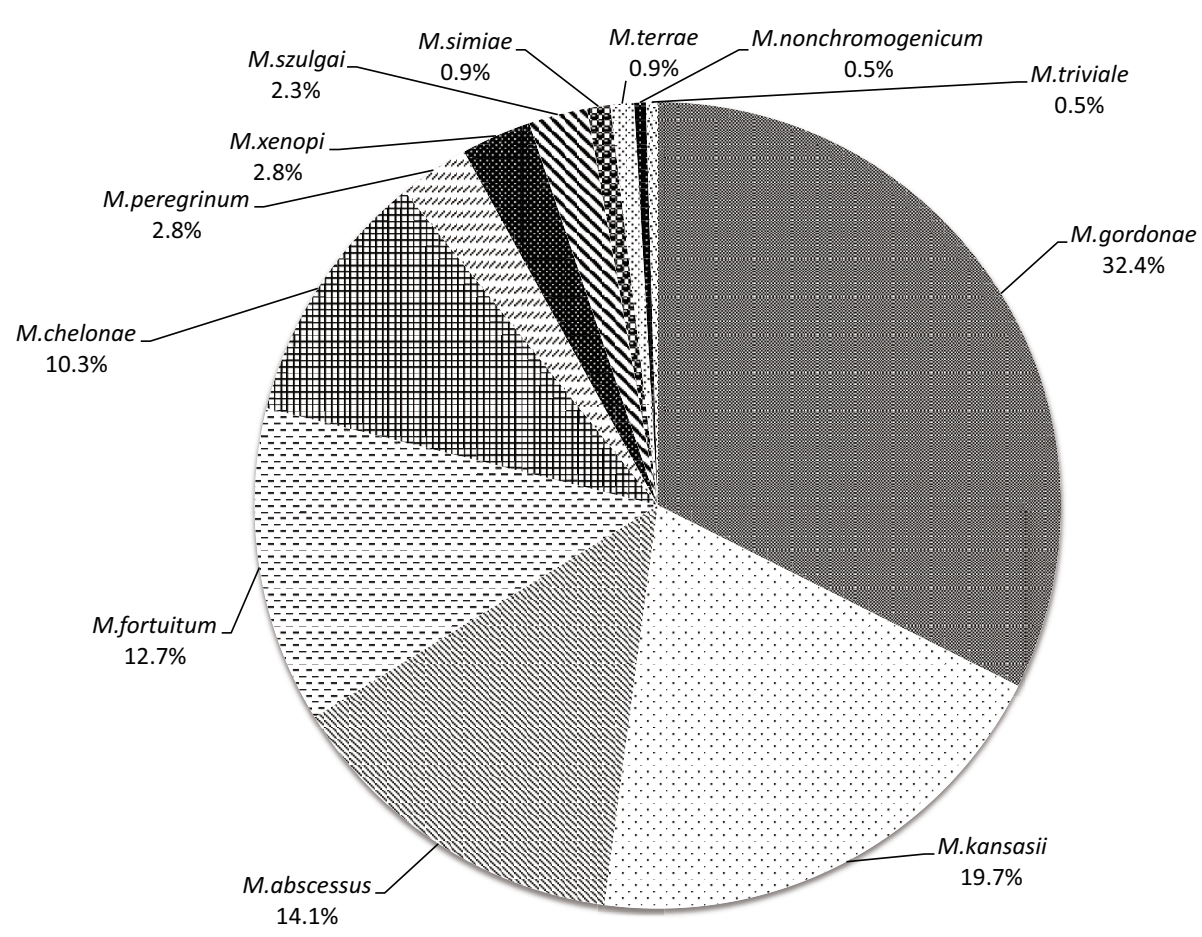

Figure. Prevalence of each nontuberculous mycobacterium isolation. The numbers of patients from whom nontuberculous mycobacteria other than Mycobacterium avium complex were isolated from respiratory specimens at least twice are shown.

Table 1. Baseline Characteristics of the 213 Patients from whom Nontuberculous Mycobacteria Other than Mycobacterium avium Complex were Isolated.

\begin{tabular}{lc}
\hline Number of patients & 213 \\
Age (years) & $68(60-76)$ \\
Male & $131(61.5 \%)$ \\
Ever smokers & $97(45.5 \%)$ \\
Previous treatment for TB & $65(30.5 \%)$ \\
BMI (mean \pm SD) $\left(\mathrm{kg} / \mathrm{m}^{2}\right)$ & $19.6(17.4-22.0)$ \\
Comorbidities & \\
Chronic obstructive lung disease & $33(15.5 \%)$ \\
Cancer & $30(14.1 \%)$ \\
Interstitial pneumonia & $22(10.3 \%)$ \\
Diabetes & $21(9.9 \%)$ \\
Bronchial asthma & $15(7.0 \%)$ \\
Chronic heart failure & $8(3.8 \%)$ \\
Collagen tissue disease & $6(2.8 \%)$ \\
Chronic pulmonary aspergillosis & $5(2.3 \%)$ \\
Symptoms & \\
Sputum & $99(46.5 \%)$ \\
Cough & $53(24.9 \%)$ \\
Hemoptysis & $13(6.1 \%)$ \\
HRCT performed at the first visit & $199(93.4 \%)$ \\
Cavity lesions on HRCT & $60(30.2 \%)$ \\
Follow-up period (months) & $70.3(34.3-99.4)$ \\
Antibiotic therapies & $87(40.8 \%)$ \\
\hline
\end{tabular}

SD: standard deviation, TB: tuberculosis, BMI: body mass index, HRCT: high-resolution computed tomography cies was detected in 7.

\section{Clinical characteristics of patients with multiple NTM isolation}

To assess the clinical significance of multiple NTM isolates in the same patient, we compared patients with multiple-species isolates to those with single-species isolates. Multiple-species isolates were more likely to be found in women than men $(58.2 \%$ vs. $31.6 \%, \mathrm{p}<0.001)$, and patients in whom multiple species were isolated were less likely to have a smoking history $(29.1 \%$ vs. $51.3 \%, \mathrm{p}=$ $0.004)$, a history of tuberculosis $(16.4 \%$ vs. $35.4 \%, \mathrm{p}=0.008)$ or cavitary lung lesions ( $16.4 \%$ vs. $32.3 \%, \mathrm{p}=0.019)$ than those with single-species isolates. Furthermore, a trend towards significance was also seen for a lower body mass index in patients with multiple NTM species isolation (multiple-species isolates $19.0 \pm 3.1$ vs. single-species isolates $20.0 \pm 3.0, \mathrm{p}=0.06$ ). No significant association was observed with age, comorbidities, follow-up duration or the administration of antibacterial agents (Table 4).

While the median number of NTM species isolated in patients with multiple isolates was two, as many as five NTM species were isolated in three patients. We investigated the clinical characteristic of these patients. Two of the three were women. The man had a smoking history and been diagnosed with chronic obstructive pulmonary disease, and the women had been diagnosed with chronic heart failure. All three patients had cavitary lung lesions. The women died during the study period. 
Table 2. Prevalence of Each Nontuberculous Mycobacterial Infection and Co-isolation of Nontuberculous Mycobacteria.

\begin{tabular}{lcc}
\hline NTM species at initial diagnosis & Total patients & Patients detected other species of NTM $(\%)$ \\
\hline M. gordonae & 69 & $24(35)$ \\
M. kansasii & 42 & $5(12)$ \\
M. abscessus & 30 & $7(23)$ \\
M. fortuitum & 27 & $7(26)$ \\
M. chelonae & 22 & $6(27)$ \\
M. peregrinum & 6 & $2(33)$ \\
M. xenopi & 6 & $2(33)$ \\
M. szulgai & 5 & $0(0)$ \\
M. simiae & 2 & $1(50)$ \\
M. terrae & 2 & $0(0)$ \\
M. nonchromogenicum & 1 & $0(0)$ \\
M. triviale & 1 & $1(100)$ \\
Total (n) & 213 & $55(26)$ \\
\hline
\end{tabular}

Data are expressed as the number $(\%)$.

*Included when different NTM species were isolated at least twice.

NTM: nontuberculous mycobacteria

\section{Discussion}

NTM species other than MAC are occasionally isolated in clinical settings. In the present study, 12 species were isolated. Most of the 213 patients enrolled in this study were middle-aged individuals with low body mass index, and almost half of them had comorbidities. More than $60 \%$ of the patients were men, with a female predominance noted for MAC lung disease (8).

In previous reports about the epidemiology of NTM lung infection, M. kansasii was the most common isolate in NTM lung disease following MAC (3-5). In our study, however, $M$. gordonae was much more frequently isolated than $M$. kansasii. M. gordonae is a free-living organism that can be frequently recovered from environmental sources, including tap water and soil (9). Generally, M. gordonae is recognized as a nonpathogenic mycobacterial contaminant. However, one global study on species diversity of NTM isolated from pulmonary samples reported that $M$. gordonae was the second-most common species after MAC in Europe and the fourth-most common in Asia (10). Although our data were obtained from a single-center study and thus might be biased, we found that over $30 \%$ of the isolates were M. gordonae. Since there are regional differences in NTM prevalence, further investigations are needed to verify whether or not $M$. gordonae is the predominant NTM isolates in our center's catchment area. Furthermore, several reports on $M$. gordonae infections have been published $(11,12)$, with only a few focusing on $M$. gordonae disease in immunocompromised hosts (13-15). In addition, the increasing proportion of elderly and immunocompromised people might result in increasing prevalence of $M$. gordonae lung disease. In fact, our results showed that the majority of patients with $M$. gordonae isolation had other comorbidities.

Multiple NTM species isolates are not rare. A previous report on M. abscessus complex (MABC) disease showed that approximately $25 \%$ of the patients had multiple NTM species, and $80 \%$ of them met the microbiological diagnostic criteria for non-MABC NTM disease (16). Our results showed some clinical characteristics for patients with multiple NTM species and several species-dependent characteristics in the patterns of co-isolation.

Our data showed that multiple NTM isolates were more common in women than men and in patients without cavity lung lesions than in those with such lesions. Lim also reported a negative association between the isolation of multiple NTM species and cavitary lung disease and suggested the possibility of having different NTM species in different ectatic bronchi or nodules (7).

The fact that a maximum of five NTM species were isolated in the same patient has some implications. All of the patients in whom five species were isolated had cavitary lung lesions and comorbidities that might result in an immunocompromised state. Co-isolation was not observed in patients with $M$. szulgai infection and was rare in patients with $M$. kansasii infection. In cases of M. abscessus infection, co-isolation was relatively common, and only MAC was co-isolated.

Several reports have suggested the clinical significance of multiple NTM species isolation $(6,17)$. Dual isolation of MAC and M. abscessus subspecies abscessus (MAA) was reported as the most common pattern of multiple NTM species isolation. A previous study also suggested that the cause of NTM species change was resistance to macrolide derived from a T/C polymorphism at the 28th nucleotide position in MAA (6). Macrolide-resistant MAA might become the main pathogen following MAC eradication with prior therapy. In fact, the present study showed that NTM species conversions were likely to occur while on therapy or within two years after therapy (6).

Despite prognostic differences among subtypes of $M$. $a b$ - 
Table 3. Details of Each Patient with Multiple Species Isolation of Nontuberculous Mycobacteria.

\begin{tabular}{|c|c|c|c|c|c|c|}
\hline $\begin{array}{l}\text { Initial NTM } \\
\text { species }\end{array}$ & $\begin{array}{l}\text { Case } \\
\text { No. }\end{array}$ & Age & Sex & $\begin{array}{c}\text { Number of } \\
\text { isolated species }\end{array}$ & $\begin{array}{l}\text { Isolation } \\
\text { pattern } * 1\end{array}$ & Co-isolated species \\
\hline \multirow{24}{*}{ M. gordonae } & 1 & 75 & Female & 2 & iii & MAC \\
\hline & 2 & 79 & Male & 2 & iii & MAC \\
\hline & 3 & 73 & Female & 2 & iii & MAC \\
\hline & 4 & 74 & Female & 2 & iii & MAC \\
\hline & 5 & 58 & Female & 2 & iii & MAC \\
\hline & 6 & 60 & Female & 2 & iii & MAC \\
\hline & 7 & 65 & Female & 2 & iii & MAC \\
\hline & 8 & 75 & Female & 2 & ii & MAC \\
\hline & 9 & 73 & Male & 2 & ii & MAC \\
\hline & 10 & 73 & Male & 2 & $\mathrm{i}$ & MAC \\
\hline & 11 & 55 & Female & 2 & $\mathrm{i}$ & MAC \\
\hline & 12 & 73 & Male & 2 & iii & M. terrae \\
\hline & 13 & 68 & Male & 2 & iii & M. chelonae \\
\hline & 14 & 79 & Male & 2 & iii & M. fortuitum \\
\hline & 15 & 77 & Female & 2 & $\mathrm{i}$ & MAC \\
\hline & 16 & 73 & Female & 3 & iii & MAC, $M$. peregrinum \\
\hline & 17 & 54 & Female & 2 & ii & MAC \\
\hline & 18 & 72 & Female & 2 & $\mathrm{i}$ & MAC \\
\hline & 19 & 62 & Female & 2 & $\mathrm{i}$ & MAC \\
\hline & 20 & 64 & Female & 2 & ii & MAC \\
\hline & 21 & 71 & Female & 3 & iii & MAC, $M$. triviale \\
\hline & 22 & 80 & Female & 3 & ii & MAC, $M$. fortuitum \\
\hline & 23 & 65 & Male & 3 & ii & MAC, $M$. fortuitum \\
\hline & 24 & 64 & Female & 5 & ii & MAC, M. abscessus, M. chelonae, M. fortuitum \\
\hline \multirow[t]{5}{*}{ M. kansasii } & 25 & 85 & Female & 2 & iii & MAC \\
\hline & 26 & 39 & Male & 2 & iii & MAC \\
\hline & 27 & 73 & Male & 2 & ii & MAC \\
\hline & 28 & 73 & Male & 2 & iii & M. gordonae \\
\hline & 29 & 53 & Female & 2 & $\mathrm{i}$ & MAC \\
\hline \multirow[t]{7}{*}{ M. abscessus } & 30 & 70 & Female & 2 & iii & MAC \\
\hline & 31 & 78 & Female & 2 & iii & MAC \\
\hline & 32 & 61 & Male & 2 & iii & MAC \\
\hline & 33 & 65 & Female & 2 & iii & MAC \\
\hline & 34 & 52 & Female & 2 & ii & MAC \\
\hline & 35 & 65 & Female & 2 & ii & MAC \\
\hline & 36 & 55 & Female & 2 & ii & MAC \\
\hline \multirow[t]{7}{*}{ M. fortuitum } & 37 & 55 & Male & 2 & iii & MAC \\
\hline & 38 & 61 & Female & 2 & iii & M. scrofulaceum \\
\hline & 39 & 68 & Female & 3 & iii & MAC, M. gordonae \\
\hline & 40 & 65 & Female & 3 & iii & M. abscessus, M. gordonae \\
\hline & 41 & 59 & Male & 2 & $\mathrm{i}$ & MAC \\
\hline & 42 & 64 & Male & 3 & ii & MAC, $M$. gordonae \\
\hline & 43 & 83 & Female & 3 & ii & MAC, M. chelonae \\
\hline \multirow[t]{6}{*}{ M. chelonae } & 44 & 67 & Male & 2 & iii & M. kansasii \\
\hline & 45 & 78 & Male & 2 & iii & MAC \\
\hline & 46 & 67 & Female & 3 & ii & MAC, M. terrae \\
\hline & 47 & 80 & Female & 3 & iii & MAC, $M$. gordonae \\
\hline & 48 & 74 & Female & 4 & iii & MAC, $M$. fortuitum \\
\hline & 49 & 60 & Female & 5 & ii & MAC, $M$. fortuitum, M. gordonae, M. szulgai \\
\hline \multirow[t]{2}{*}{ M. peregrinum } & 50 & 76 & Male & 2 & iii & MAC \\
\hline & 51 & 74 & Female & 2 & ii & MAC \\
\hline \multirow[t]{2}{*}{ M. xenopi } & 52 & 64 & Male & 5 & ii & MAC, M. chelonae, M. fortuitum, M. gordonae \\
\hline & 53 & 70 & Male & 2 & ii & M. nonchromogeni \\
\hline M. simiae & 54 & 78 & Male & 2 & iii & MAC \\
\hline M. triviale & 55 & 22 & Male & 3 & ii & MAC, M. abscessus \\
\hline
\end{tabular}

*1 Patterns of multiple NTM isolation were categorized into three groups: (i) isolation of multiple NTM species simultaneously $(\mathrm{A}+\mathrm{B} \rightarrow \mathrm{A}+\mathrm{B})$, (ii) alternating isolation of multiple NTM species $(\mathrm{A} \rightarrow \mathrm{B} \rightarrow \mathrm{A} \rightarrow \mathrm{B})$, (iii) disappearance of initial NTM species and detection of new NTM species $(\mathrm{A} \rightarrow \mathrm{A} \rightarrow \mathrm{B} \rightarrow \mathrm{B} \rightarrow \mathrm{B})$. We included all patterns of multiple NTM isolation.

NTM: nontuberculous mycobacteria, MAC: Mycobacterium avium complex 
Table 4. Results of a Comparison Analysis between the Multiple-species Isolation Group and the Single-species Isolation Group $(\mathrm{n}=\mathbf{2 1 3})$.

\begin{tabular}{lccc}
\hline \multicolumn{1}{c}{ Clinical characteristics } & $\begin{array}{c}\text { multiple isolate } \\
(\mathrm{n}=55)\end{array}$ & $\begin{array}{c}\text { single isolate } \\
(\mathrm{n}=158)\end{array}$ & p value \\
\hline Age (years) & $70(63.5-74)$ & $67(57-76)$ & 0.562 \\
Female & $32(58.2 \%)$ & $50(31.6 \%)$ & $<0.001$ \\
Ever smoker & $16(29.1 \%)$ & $81(51.3 \%)$ & 0.004 \\
Previous treatment for TB & $9(16.4 \%)$ & $56(35.4 \%)$ & 0.008 \\
BMI (mean \pm SD) $\left(\mathrm{kg} / \mathrm{m}^{2}\right)$ & $19.0 \pm 3.1$ & $20.0 \pm 3.0$ & 0.063 \\
Comorbidities & & & \\
Chronic obstructive lung disease & $7(12.7 \%)$ & $26(16.5 \%)$ & 0.5 \\
Cancer & $6(10.9 \%)$ & $24(15.2 \%)$ & 0.423 \\
Interstitial pneumonia & $3(5.5 \%)$ & $19(12.0 \%)$ & 0.164 \\
Diabetes & $3(5.5 \%)$ & $18(11.4 \%)$ & 0.199 \\
Bronchial asthma & $2(3.6 \%)$ & $13(8.2 \%)$ & 0.248 \\
Chronic heart failure & $3(5.5 \%)$ & $5(3.2 \%)$ & 0.447 \\
Collagen tissue disease & $4(7.3 \%)$ & $2(1.3 \%)$ & 0.021 \\
Chronic pulmonary aspergillosis & $2(3.6 \%)$ & $3(1.9 \%)$ & 0.468 \\
Symptoms & & & \\
Sputum & $23(41.8 \%)$ & $76(48.1 \%)$ & 0.331 \\
Cough & $11(20.0 \%)$ & $42(26.6 \%)$ & 0.421 \\
Hemoptysis & $2(3.6 \%)$ & $11(7.0 \%)$ & 0.375 \\
Cavity lesions on HRCT & $9(16.4 \%)$ & $51(32.3 \%)$ & 0.019 \\
Follow-up period (months) & $67.0(34.9-99.3)$ & $72.2(33.6-99.2)$ & 0.857 \\
Antibiotic therapies & $20(36.4 \%)$ & $67(42.4 \%)$ & 0.432 \\
\hline SD stand & & &
\end{tabular}

SD: standard deviation, TB: tuberculosis, BMI: body mass index, HRCT: high-resolution computed tomography

scessus, we did not identify any in our study, and only MAC was isolated in patients co-infected with MAA. In some of our cases, alternate isolation of MAC and M. $a b$ scessus occurred. Alternate isolation may be the result of bacterial competition while on the medication. Since there is no established therapy for both MAC and MAA, their coisolation poses a treatment challenge.

Another important point in our study was that no other NTM species were isolated in patients with $M$. szulgai infection. The clinical characteristics of $M$. szulgai infection are believed to be similar to those of $M$. kansasii infection, and its biological life cycle resembles that of $M$. malmoense (18). For this reason, co-isolation was relatively infrequent in patients with M. kansasii infection in our study.

Likewise, other NTMs were not isolated in patients with $M$. terrae infection or $M$. nonchromogenicum infection. $M$. terrae and $M$. nonchromogenicum are grouped together as $M$. terrae complex. It is interesting that these species, classified in the same group, showed the same isolation pattern, never being co-isolated with other NTMs. Nevertheless, since only 3 of the 213 patients were identified as having $M$. terrae complex in the present study, concluding that $M$. terrae complex infection had a similar clinical course to $M$. szulgai infection may be inappropriate.

Several limitations associated with the present study warrant mention. First, this was a retrospective single-center study, so our results may not be generalizable to other settings. Second, our hospital does not routinely distinguish be- tween $M$. avium and $M$. intracellulare, and the isolation rates of these two species are not available. Third, detailed information on medications and disease severities were not explored. We included all co-isolates even after treatment initiation.

As stated previously, the increase in numbers of elderly and immunocompromised patients may affect the distribution of NTM species. Further studies with larger sample sizes and a longer follow-up are needed to conclude the clinical significances.

\section{Conclusion}

We evaluated 12 NTM species other than MAC isolated at least twice and described the clinical characteristics of patients with multiple NTM species isolates. Multiple NTM species isolates were common, observed in $26 \%$ of nonMAC NTM-isolated patients, and the patterns of co-isolation depended on the NTM species. Since multiple NTM species isolates are not rare, regular sputum culture monitoring should be performed during and after treatment of NTM infection.

The authors state that they have no Conflict of Interest (COI).

\section{References}

1. Namkoong H, Kurashima A, Morimoto K, et al. Epidemiology of 
pulmonary nontuberculous mycobacterial disease, Japan. Emerging Infect Dis 22: 1116-1117, 2016.

2. Kimizuka Y, Hoshino Y, Nishimura T, et al. Retrospective evaluation of natural course in mild cases of Mycobacterium avium complex pulmonary disease. PLoS One 14: 1-18, 2019.

3. Morimoto K, Hasegawa N, Izumi $\mathrm{K}$, et al. A laboratory-based analysis of nontuberculous mycobacterial lung disease in Japan from 2012 to 2013. Ann Am Thorac Soc 14: 49-56, 2017.

4. Namkoong H, Kurashima A, Morimoto K, et al. Epidemiology of pulmonary nontuberculous mycobacterial disease, Japan. Emerg Infect Dis 22: 1116-1117, 2016.

5. Diel R, Ringshausen F, Richter E, et al. Microbiological and clinical outcomes of treating non-Mycobacterium avium complex nontuberculous mycobacterial pulmonary disease: a systematic review and meta-analysis. Chest 152: 120-142, 2017.

6. Lee JS, Lee JH, Yoon SH, et al. Implication of species change of nontuberculous mycobacteria during or after treatment. BMC Pulm Med 17: 213, 2017.

7. Lim HJ, Park CM, Park YS, et al. Isolation of multiple nontuberculous mycobacteria species in the same patients. Int J Infect Dis 15: e795-e798, 2011.

8. Hagiwara E, Katano T, Isomoto K, et al. Clinical characteristics and early outcomes of patients newly diagnosed with pulmonary Mycobacterium avium complex disease. Respir Investig 57: 54-59, 2019.

9. Griffith DE, Aksamit T, Brown-Elliott BA, et al. An official ATS/ IDSA statement: diagnosis, treatment, and prevention of nontuberculous mycobacterial diseases. Am J Respir Crit Care Med 15 367-416, 2007.

10. Hoefsloot W, van Ingen J, Andrejak C, et al. The geographic diversity of nontuberculous mycobacteria isolated from pulmonary samples: an NTM-NET collaborative study. Eur Respir J 42: 1604-1613, 2013.
11. Nakazawa A, Hagiwara E, Ikeda $S$, Oda $T$, Komatsu S, Ogura $T$. A case of pulmonary Mycobacterium gordonae infection diagnosed by gastri juice culture and successfully treated with multidrug chemotherapy. Kekkaku 87: 727-731, 2012.

12. Fujita $Y$, Matsumoto $H$, Fujikane $T$, et al. Pulmonary infection caused by Mycobacterium gordonae in immunocompetent patient. Kekkaku 75: 369-374, 2000.

13. Youssef D, Shams WE, Elshenawy Y, El-Abbassi A, Moorman JP. Pulmonary infection with caseating mediastinal lymphadenitis caused by Mycobacterium gordonae. Int J Mycobacteriol 3: 220223, 2014.

14. Fujita $\mathrm{Y}$, Matsumoto H, Fujikane $\mathrm{T}$, et al. Pulmonary infection caused by Mycobacterium gordonae in immunocompetent patient. Kekkaku 75: 369-374, 2000.

15. Aguado JM, Gómez-Garcés JL, Manrique A, Soriano F. Pulmonary infection by Mycobacterium gordonae in an immunocompromised patient. Diagn Microbiol Infect Dis 7: 261-263, 1987.

16. Morimoto K, Nakagawa T, Asami T, et al. Clinico-microbiological analysis of 121 patients with pulmonary Mycobacteroides abscessus complex disease in Japan - an NTM-JRC study with RIT. Respir Med 145: 14-20, 2018.

17. Griffith DE, Philley JV, Brown-Elliott BA, et al. The significance of Mycobacterium abscessus subspecies abscessus isolation during Mycobacterium avium complex lung disease therapy. Chest 147: 1369-1375, 2015.

18. Sekine A, Hagiwara E, Ogura T, et al. Four cases of pulmonary infection due to Mycobacterium szulgai with a review of previous case reports in Japan. Nihon Kokyuki Gakkai Zasshi 46: 880-888, 2008.

The Internal Medicine is an Open Access journal distributed under the Creative Commons Attribution-NonCommercial-NoDerivatives 4.0 International License. To view the details of this license, please visit (https://creativecommons.org/licenses/ by-nc-nd/4.0/).

(C) 2021 The Japanese Society of Internal Medicine Intern Med 60: 3213-3219, 2021 